\title{
PRÁCTICAS SOCIALES EN TORNO A LA INCLUSIÓN \\ DE LA DISCAPACIDAD SENSORIAL \\ EN ESCUELAS PÚBLICAS DE COPIAPÓ DE CHILE
}

\section{Social practices in the context of sensorial disabilities inclusion in the public schools of Copiapó Chile}

\begin{abstract}
FRANCISCO LERÍA DULČIĆ”, JORGE SALGADO ROA*
INGEBORG ALMONTE OSSANDÓN, MARÍA VEGA TORRES y MARISOL VÉLIZ GONZÁLEZ $^{* *}$
\end{abstract}

Resumen. El objetivo del estudio es describir las prácticas sociales que el Estado, las escuelas y familias elaboran para abordar la inclusión de la discapacidad sensorial visual y auditiva en el contexto educativo de la ciudad de Copiapó. Participaron expertos en temáticas de inclusión sensorial, profesores y familias de niños y jóvenes con alguna discapacidad sensorial. Se realizó desde un enfoque cualitativo de tipo descriptivo, incorporando algunas de las fases analíticas propuestas por el método de la Teoría Fundamentada, utilizando

\footnotetext{
* Psicólogo, Magister en Psicología Clínica por la Universidad de Zagreb, Croacia. Docente e investigador del Departamento de Psicología de la Universidad de Atacama, Copiapó, Chile. Sus áreas de interés son la inclusividad educativa, convivencia escolar, lenguaje, educación emocional y educación contemplativa.

${ }^{* *}$ Psicólogo, Doctor en Ciencias de la Educación por la Universidad de la Serena, Chile. Docente e investigador del Departamento de Psicología de la Universidad de Atacama, Copiapó, Chile. Sus áreas de interés son la inclusión y vulnerabilidad escolar, calidad de vida laboral y bienestar en el contexto organizacional, y validación de instrumentos psicométricos.

*** Licenciadas en Psicología de la Universidad de Atacama, Copiapó, Chile. Sus áreas de interés son la psicología educacional y comunitaria.
} 
entrevistas, observaciones participantes y análisis de documentos. Se concluyó que los informantes claves consideran la inclusión educativa deficiente en lo que respecta a los recursos materiales, acceso físico al establecimiento, recursos técnicos, humanos e infraestructura de los dispositivos educacionales. De esta manera existiría una distancia entre lo que declara el Estado chileno (leyes y decretos) y sus planes y programas, así como también entre lo que realiza la unidad educativa del establecimiento y lo que entienden las familias por inclusión al sistema educativo.

Palabras claves: Integración escolar, necesidades educativas especiales, discapacidad, prácticas inclusivas y déficit sensorial

Abstract. The aim of the study is to describe the social practices that the state, schools and families elaborate to address the inclusion of visual and auditory sensory disabilities in the educational context of the city of Copiapó. Participants included experts on topics of sensory inclusion, teachers and families of children and young people with a sensory impairment. The study was conducted from a qualitative descriptive approach that incorporate some of the elements proposed by the method of the Grounded Theory, through interviews, participant observation and document analysis. The key informants considered the educational inclusion to be poor with regard to the material resources, physical access to the school facilities, technical, human and infrastructure resources of the educational devices. By this information it was concluded there is a gap between what the Chilean State had declared about sensorial inclusion by their laws and decrees, and what the educational institution and the experts and the families actually understand and finally do for inclusion in the educational system. 
Prácticas sociales en torno a la inclusión de la discapacidad sensorial en escuelas públicas de Copiapó de Chile

Keywords. School integration, special educational needs, disability, inclusive practices and sensory deficits

\section{INTRODUCCIÓN}

La educación en Chile ha comenzado a incursionar hace ya un tiempo en la promoción de la igualdad e inclusión con el fin de asegurar el derecho a una educación de calidad para todos, particularmente para aquellos que muestran algún tipo de discapacidad. Según la Declaración Universal de los Derechos del Niño, aprobada por la Asamblea General de las Naciones Unidas el 20 de Noviembre de 1959:

El niño [y el adolescente] tiene derecho a recibir educación, que será gratuita y obligatoria por lo menos en las etapas elementales. Se le dará una educación que favorezca su cultura general y le permita, en condiciones de igualdad de oportunidades, desarrollar sus aptitudes y su juicio individual, su sentido de responsabilidad moral y social y llegar a ser un miembro útil de la sociedad. (ONU, 1959, párr.8)

En esta dirección, "en Chile durante los últimos tres años ha habido un avance y consolidación producto de varias iniciativas conjuntas de SENADIS (Secretaría Nacional por los Derechos de las Personas con Discapacidad); y un número importante de instituciones de educación superior" (Lissi, Zuzulich, Hojas, Achiardi, Salinas \& Vasquez, 2014, p.18). Las políticas específicas de integración del Ministerio de Educación Chileno (en adelante MINEDUC) han permitido que las instituciones educativas incorporen a los niños que muestran algún tipo de discapacidad en los establecimientos educacionales públicos y subvencionados del país, comenzando ya desde 1990 con el Decreto $\mathrm{N}^{\circ} 490^{1}$, que facilitó la integración en las aulas regulares de los niños con algún tipo de discapacidad.

Sin embargo, este proceso no ha sido implementado de forma homogénea a lo largo del país. Por ejemplo, la realidad en la ciudad de Copiapó, ha mostrado ser altamente deficiente en lo que refiere a la gestión y atención a la discapacidad,

\footnotetext{
${ }^{1}$ Este Decreto fue derogado posteriormente por el Decreto Supremo № 1 del MINEDUC (1998).
} 
Prácticas sociales en torno a la inclusión de la discapacidad sensorial en escuelas públicas de Copiapó de Chile

situándose esta ciudad y sus autoridades en el ranking de las 20 municipalidades cuestionadas por la Contraloría debido a la tardía implementación de los programas educativos que favorecen la inclusión e integración de estudiantes con Necesidades Educativas Especiales (en adelante NEE). Un estudio realizado por el Centro de Investigación Periodística Ranking de las 20 municipalidades más cuestionadas por la Contraloría, constata que:

Una de las primeras observaciones que establece la Contraloría Regional de Atacama respecto al uso de los recursos SEP (Subvención Escolar Preferencial) recibidos por la llustre Municipalidad de Copiapó, es la dificultad que tuvieron para obtener la información contable para la auditoría. De hecho, luego de solicitar reiteradamente los antecedentes (...) la Contraloría hace hincapié en el desorden administrativo del Departamento de Educación de este municipio. (Guerra, Arcos, Riquelme, Ramírez \& Guzmán, 2012, párr. 1 y 2)

Por otra parte, la región de Atacama presenta un menor número de Proyectos de Integración Escolar (en adelante PIE) por escuela, existiendo diferencias notorias con otras regiones del país; por ejemplo, la Cuarta Región de Coquimbo posee centros educativos para la discapacidad sensorial, incluso escuelas regulares que ofrecen una gama de posibilidades de integración. Por el contrario, en la región de Atacama y su capital regional de la ciudad de Copiapó, se ha constatado que no existe diversidad de oferta, particularmente en la educación secundaria donde los establecimientos con integración son escasos. Además, las carencias y faltas en la atención de estudiantes con NEE se ven intensificadas con el desconocimiento de la materia y sus derechos por parte de los directivos de los centros educativos regionales, los cuales - por ejemplo- no postulan a los beneficios de la ley.

Otra debilidad percibida en la región es la falta de continuidad entre los distintos procesos de intervención en los ciclos educativos, debido a que los profesionales trabajadores del PIE y equipos conformados por la SEP no comienzan en los plazos de ingreso a los sistemas educativos asignados por el MINEDUC. El Municipio de Copiapó realiza contrataciones tardías, generando un 
Prácticas sociales en torno a la inclusión de la discapacidad sensorial en escuelas públicas de Copiapó de Chile

proceso lento tanto en la evaluación como en la intervención de estos niños, niñas y jóvenes. Como lo señala la revista Atacama Viva, donde la Dirección Regional de Superintendencia de Educación sanciona al DAEM (Departamento de Administración de Educación Municipal) de Copiapó:

Ante la denuncia presentada del Colegio de Profesores realizada el 17 de diciembre pasado, se desarrolló un proceso investigativo de parte de la Superintendencia de Educación Escolar sobre el Departamento de Educación Municipal de Copiapó. Los temas denunciados en su momento fueron el no pago de imposiciones y el no pago del refuerzo educativo. (Castillo, 2013, párr.2)

Considerando esta situación que hace de la discapacidad sensorial un tema descuidado a nivel regional y comunal, se plantea el presente estudio de carácter cualitativo iniciado por los siguientes cuestionamientos: ¿Cuáles son las prácticas sociales de inclusión de la discapacidad sensorial que se implementan en los sistemas educativos de la comuna de Copiapó? ¿Cuáles son las prácticas sociales que define el Estado en torno a la discapacidad sensorial? ¿Cómo se implementan los programas de integración en las escuelas regulares de Copiapó? ¿Cuáles son las interpretaciones que la escuela y las familias generan en torno a la inclusión de la discapacidad sensorial?

\section{DISCAPACIDAD Y EDUCACIÓN}

El concepto de discapacidad a lo largo de la historia ha sufrido grandes transformaciones. Los movimientos sociales del siglo pasado fueron los propulsores de los cambios que experimentaría, originados en la demanda social por más igualdad de derechos entre hombres y mujeres sin importar la raza, edad, género o cultura (Marchesi, Coll \& Palacios, 2012). A partir del año 2001, entra en vigencia la Clasificación Internacional del Funcionamiento de la Discapacidad y de la Salud (CIF) que propone una nueva clasificación que no considera las consecuencias de una enfermedad, sino que se habla de funcionamiento, discapacidad y salud (Egea y Sarabia, 2001). Al mismo tiempo este nuevo marco 
Prácticas sociales en torno a la inclusión de la discapacidad sensorial en escuelas públicas de Copiapó de Chile

conceptual le otorga amplitud y un carácter más técnico y de naturaleza clínica al fenómeno de la discapacidad. Además, se empezó a contar con técnicas de evaluación psicométricas, que permitieron la especialización y tecnificación del término (Marchesi et al., 2012).

El Informe Mundial sobre la Discapacidad del año 2013 afirma que la educación contribuye a la formación de capital humano y, por lo tanto, es uno de los principales factores determinantes del bienestar y la prosperidad de las personas. Siendo la exclusión de las personas con discapacidad de oportunidades de educación y empleo un hecho de elevado costo social y económico, se señala que los gobiernos deben preocuparse y procurar la inclusión de estos individuos en el sistema educativo regular con los apoyos técnicos y profesionales necesarios. En este sentido, la OMS en el año 2011 realizó un estudio a través del cual fueron revisadas las políticas educativas inclusivas en 28 países que implementaron la iniciativa "Vía Rápida de Educación para Todos". Se observó que 18 de estos países dieron muy poca o nada de información sobre estrategias para incluir a los niños y niñas con discapacidad en el sistema educativo, descubriéndose que: "un déficit habitual en las políticas educativas es la falta de incentivos económicos y de otro tipo orientados a que los niños y niñas con discapacidad acudan a la escuela, así como la falta de servicios de apoyo y protección" (OMS, 2011, p. 9).

Para subsanar en parte esta situación, la Asamblea Mundial de la Salud ha promovido un paquete de estrategias de prevención, llamado "Plan de Acción para la Prevención de la Ceguera y la Discapacidad Visual", orientado a disminuir la discapacidad visual en un $25 \%$ para el año 2019 , considerando que el $80 \%$ de los casos de discapacidad visual son prevenibles o curables (OMS, 2011). Otro de los esfuerzos por introducir el concepto y la práctica de la inclusión en el contexto educativo han sido los impulsos de los gobiernos por integrar, desde un punto de vista de los derechos humanos, el equitativo acceso a la educación no segregada y discriminante para estudiantes con discapacidad. Esto requirió planes reguladores que manejen los recursos para solventar programas y también 
Prácticas sociales en torno a la inclusión de la discapacidad sensorial en escuelas públicas de Copiapó de Chile

políticas de Estado que faciliten la integración de planes en las aulas educativas. En este sentido se señala:

La inclusión no solo trata de captar la presencia física de los estudiantes etiquetados como niños con necesidades educativas especiales, con o sin discapacidad, sino que busca satisfacer las necesidades de todos los alumnos (...); para ello será necesario respetar sus diferencias y promover el desarrollo de sus habilidades cognoscitivas y de comunicación junto con sus pares en el aula regular. (Garnique-Castro \& Gutiérrez-Vidrio, 2012, p. 580)

Un último aspecto a destacar es el interés del sistema educativo por la construcción de un currículo escolar respetando las necesidades de aprendizaje de cada estudiante y su perfil propio y distintivo, transitando, como la afirma Ainscow (2005), desde un concepto de inclusividad educativa desde las NEE a ampliarse a todas las necesidades de desarrollo humano. Al respecto, García (2011) señala:

$\mathrm{Si}$ implementamos un currículo capaz de direccionar todos los esfuerzos hacia el desarrollo de las competencias de cada persona, donde su desempeño permita utilizar los recursos existentes, materiales y tecnológicos, físicos e intelectuales, cognitivos y emocionales de manera óptima y racional, capaces de potenciar al máximo la dimensión humana, capaz de conocer, interpretar y transformar la realidad, lo que implica estimular la creatividad, la imaginación, el pensamiento divergente. (p. 2)

De esta forma, estos autores destacan la importancia y la necesidad de construir un curriculum inclusivo que incorpore el desarrollo de competencias desde una perspectiva amplia e integral, alineado con las tendencias actuales y planteadas originalmente para el mundo educativo en el informe Delors (1996).

Una mirada a la literatura especializada destaca una serie de estudios que aportan al entendimiento y contextualización del concepto de inclusividad, diversidad y discapacidad sensorial, desde el punto de vista de las prácticas sociales y los factores que la promueven. Por ejemplo, en España se investigó en una muestra de 120 maestros de educación primaria, encargados de la asignatura 
Prácticas sociales en torno a la inclusión de la discapacidad sensorial en escuelas públicas de Copiapó de Chile

"Atención a la diversidad", sus perspectivas, pensamientos e ideas respecto de la diversidad e inclusión. El artículo sugiere que: "una de las formas de avanzar en el proceso de atender a la diversidad, desde una perspectiva inclusiva, es la investigación y reflexión de los futuros profesores en las temáticas de interés" (Vigo \& Soriano, 2014, p. 40). Este estudio destaca el trabajo colaborativo y participativo del profesorado como principal factor de cambio y apertura de la escuela, tanto en lo referente a la gestión escolar como a la transformación de la práctica educativa en el sentido de una mayor riqueza e inclusividad. Otro estudio ha señalado la relevancia y papel activo de las prácticas de enseñanza dinámicas, vistas como más efectivas para el cumplimiento de los objetivos de aprendizaje, generando en los actores del proceso educativo mayor participación y compromiso (Camacho, 2011).

Un aspecto importante a resaltar en la literatura, es la actitud de los profesores hacia la inclusión educativa y los factores que les impactan frente a ella. Por ejemplo, se ha visto que la experiencia directa en el tema de interés, las características específicas y particulares de los estudiantes con discapacidad, el tiempo dedicado al trabajo directo con ellos, la disponibilidad de recursos de apoyo y la capacitación docente en el tema -entre otros factores- tienen un directo impacto en la actitud del profesorado frente a la inclusión educativa de niños y niñas con NEE. Al docente se le destaca según sus actitudes y disposiciones personales y profesionales como un agente que "puede constituirse en una barrera o un agente facilitador de las prácticas inclusivas" (Granada, Pómez \& Sanhueza, 2013, p. 57). Un buen ejemplo de ello son las necesidades de los estudiantes ciegos. Más allá de los aspectos técnicos (apoyo tecnológico) se necesita, además de brindarle a una persona ciega el acceso apropiado a las instalaciones, "proveerle de un buen trato del personal docente y administrativo de manera que se equipare (acción de nivelación) del trato que reciben todos los alumnos" (Romero, Kitaoka \& Rodríguez, 2014, p.5).

Se ha destacado en estos aspectos el ejemplo que la educación parvularia ha entregado en torno a la inclusión, en un sentido general, amplio e integral, la ha 
Prácticas sociales en torno a la inclusión de la discapacidad sensorial en escuelas públicas de Copiapó de Chile

entendido, no sólo como una respuesta a las necesidades específicas y particulares de los estudiantes con discapacidad, sino como una respuesta global a todos aquellos estudiantes que se encuentren en situaciones difíciles con algún tipo de necesidad. Además, dando un especial énfasis a la formación de educadoras con actitudes abiertas a la diversidad, con una mayor capacidad de acogida y responsabilidad, y mejor capacitadas en educación inclusiva al interior de los jardines infantiles. Desde estas experiencias se concluye "la importancia de una adecuada formación técnica de las educadoras para facilitar la apertura y disposición por la inclusión al interior de los jardines infantiles del país" (Larraguibel, 2012, p.86).

La inclusión escolar es un tema cada vez más relevante en el sistema educativo chileno y han sido los PIE quienes han permitido una mayor cobertura de atención especializada. El Centro de Innovación en la Educación de la Fundación Chile realizó un estudio respecto a la implementación de programas escolares en establecimientos educacionales que cuentan con Proyectos de Integración a nivel nacional. Sus hallazgos muestran la existencia de un incremento de niños con necesidades educativas especiales en las escuelas ordinarias y especiales, contando desde el año 2009 con un número de estudiantes integrados en las escuelas subvencionadas de 68.117 a 171.884 en el año 2012; y de un número preliminar de 210.332 para el año 2013. También se encontró que las escuelas que participan en el PIE han disminuido en número, "de 5.574 en el año 2010 a 4.626 en el año 2013" (Fundación Chile, 2013, p.21). No obstante, se debe notar que en el país existen diferencias entre el ámbito municipal y particular. Los establecimientos municipales poseen una cantidad de niños con NEE mucho mayor que el sector particular. Las cifras indican que, por ejemplo, "en $1^{\circ}$ básico las escuelas municipales abarcan no sólo una mayor cantidad de estudiantes con NEE, sino también una mayor cantidad y diversidad en los diagnósticos de discapacidad que son parte de la subvención estatal chilena" (Torres, 2013, p.137). 
Prácticas sociales en torno a la inclusión de la discapacidad sensorial en escuelas públicas de Copiapó de Chile

A partir de la Ley 20.422, pronunciada en Chile el año 2013, se estableció una agenda para trabajar durante siete años a partir del Plan Nacional de Acción para la Inclusión Social de las personas con discapacidad, que fue elaborado por el Servicio Nacional de la Discapacidad (SENADIS). Este plan de acción tenía como objetivo entregar al Estado un instrumento de gestión pública en materia de discapacidad con el objetivo general de:

Velar por el acceso de las personas con discapacidad a un sistema educativo inclusivo y con igualdad de oportunidades en todos sus niveles, desde la educación parvularia a la educación superior, caracterizado por la innovación, adecuaciones curriculares y de infraestructura, con personal docente concienciado, capacitado y formado profesionalmente en la educación inclusiva. (2013, p. 18)

En los lineamientos estratégicos de la política de educación generados por esta Ley, se consideran temas relacionados con la inclusión como: discapacidad en el sistema general de educación; promoción e implementación de un diseño universal de educación inclusiva; eliminar del sistema educativo toda forma de discriminación; establecer programas de coordinación con instituciones y entidades públicas o privadas para apoyo integral de las familias; y prestar apoyo en la atención escolar para estudiantes con patologías o condiciones médicas ya sea en centros hospitalarios o domicilios.

El informe de resultados emitido por el Sistema de Medición de la Calidad de la Educación (SIMCE), en el año 2011, incorporó en su medición estudiantes que presentan discapacidades sensoriales de cuarto básico de educación primaria provenientes de las regiones de Valparaíso, Biobío y Metropolitana. Con el fin de tener una visión real sobre los aprendizajes logrados y la necesidad de avanzar en cambios curriculares o nuevas reformas en el área, se evaluaron 132 estudiantes que representan el $87 \%$ de aquellos con discapacidad sensorial provenientes de 79 establecimientos regulares; y nueve establecimientos especiales (Sistema de Medición de la Calidad de la Educación, 2011). Los resultados muestran que existe una menor brecha entre niños con discapacidad visual y aquellos sin 
Prácticas sociales en torno a la inclusión de la discapacidad sensorial en escuelas públicas de Copiapó de Chile

discapacidad sensorial. De acuerdo a estos resultados, las gestiones realizadas por los gobiernos han permitido acotar la brecha entre los establecimientos especiales y establecimientos regulares, indicando que los planes y programas han potenciado las habilidades y conocimientos de los niños con NEE, mejorando su rendimiento escolar. No obstante, si bien actualmente existen medidas para la inclusión de los estudiantes con discapacidad, se ha detectado que mientras más aumenta la edad de los mismos, mayor es la exclusión que experimentan.

Ocampo (2011) reflexiona respecto a los desafíos que en este cometido los gobiernos y sus políticas públicas deben enfrentar. En un estudio conducido con el objetivo de precisar y conocer los factores que promueven y restringen el desarrollo de una formación profesional de calidad para los estudiantes con discapacidad de educación superior, se investigaron las perspectivas de docentes y estudiantes de dos universidades privadas de Santiago. Este concluye que "las principales barreras a la inclusión están puestas en el ejercicio desigual del derecho a la educación, el cual se concretiza sólo cuando se permite que los alumnos en situación de discapacidad ingresen a la universidad" (p. 238).

\section{MATERIALES Y MÉTODO}

La presente investigación utiliza un diseño cualitativo. Se basa en los postulados de Strauss y Corbin (2002) y Charmaz (2007) en relación a un "trabajo de investigación más enraizado en la descripción interpretativa que en la construcción de teoría formal emergente; en el cual el investigador adopta un papel importante en el proceso de indagación" (p. 20). Se utilizan algunas fases del proceso analítico planteadas por el método de la Teoría Fundamentada, definida como "una metodología de análisis de información que propone un procedimiento de actuación en el trabajo con los datos mediante la aplicación de un método, que los autores denominan, método comparativo constante" (Trinidad, Carrero \& Soriano, 2006, p.16). 
Prácticas sociales en torno a la inclusión de la discapacidad sensorial en escuelas públicas de Copiapó de Chile

El análisis se efectuó de acuerdo a las siguientes etapas (Strauss \& Corbin, 2002): a) Codificación abierta: Los textos se sintetizan en categorías conceptuales que aparecen contextualmente y no se determinan con anterioridad, de acuerdo al procedimiento de la Teoría Fundamentada; b) Codificación axial (familias de códigos): los códigos se agrupan en familias que se determinan de acuerdo a ciertos criterios de campos semánticos; y c) Descripción de las familias de códigos: se describieron las principales características del objeto de estudio de acuerdo a la agrupación en familias de códigos. Para el análisis se utilizó el software Atlas/Ti versión 7.0 que permite reducir los datos cualitativos siguiendo los pasos mencionados.

La muestra de informantes-participantes en este estudio se constituye por: a) expertos en temáticas de inclusión sensorial con experiencia de trabajo con niños y jóvenes con discapacidad visual y/o auditiva; b) profesores que se desempeñen en establecimientos educacionales de tipo municipal adscrito al Proyecto de Integración Escolar (PIE); y c) familias de niños y jóvenes con alguna discapacidad visual y/o auditiva.

\section{Técnicas de recolección de datos}

En cuánto a las técnicas de recolección de datos, se utilizaron las siguientes:

ENTREVISTAS. Entrevistas no estructuradas inicialmente con un guión temático orientador. Se realizaron 21 entrevistas desglosadas en: cinco expertos, nueve docentes y siete familias (jóvenes y padres). La información generada en estas instancias fue digitalizada y transcrita constituyendo un corpus único, consignando todos aquellos datos sensibles que pudieran identificar a alguno de los participantes. La conformidad de los participantes fue registrada digitalmente y en papel al inicio de cada entrevista.

OBSERVACIÓN PARTICIPANTE. Se observaron en el espacio escolar los dispositivos educativos que generan la inclusión de niños con discapacidad visual y/o auditiva, con la finalidad de conocer el contexto en el que se desarrollan las 
Prácticas sociales en torno a la inclusión de la discapacidad sensorial en escuelas públicas de Copiapó de Chile

prácticas inclusivas y comprender las narraciones de los informantes claves en el momento de enunciar la temática estudiada. La observación participante fue desarrollada de manera no sistemática y no encubierta (Iñiguez, 1999), con el objetivo de recopilar información de los elementos semióticos de las prácticas sociales asociadas, como el lenguaje no verbal, dando lugar a la redacción del Diario de campo para notas descriptivas que aporten lo que dicen las personas implicadas (interpretaciones, vivencias y reflexiones personales sobre el análisis de lo observado). Se realizaron un total de diez observaciones en tres establecimientos educacionales.

ANÁLISIS DE DOCUMENTOS. Se analizaron seis documentos provenientes del Estado de los cuales dos son leyes (Ley de discapacidad 19.284 y Ley 20.422), tres decretos (Decreto $N^{\circ} 89, N^{\circ} 815$ y $N^{\circ} 170$ ) y un documento emanado del PIE.

CONSIDERACIONES ÉTICAS. La participación fue voluntaria; una vez otorgado el permiso por parte de los directivos de los establecimientos se dio a conocer los objetivos del estudio y el carácter confidencial de las entrevistas y observaciones. Posteriormente se solicitó la firma de un consentimiento informado por parte de docentes, alumnos y apoderados.

\section{RESULTADOS}

El relato de los informantes, así como las observaciones de campo y el análisis de leyes y decretos, muestran en su conjunto que la inclusión al interior de los establecimientos educacionales de la comuna de Copiapó es visible y está presente en las acciones y discursos de sus actores principales. No obstante, en estas acciones se observan carencias en relación a su comprensión, entendimiento y alcances. A continuación, se destacan las agrupaciones semánticas más relevantes obtenidas tras la codificación axial.

\section{Prácticas sociales inclusivas de las escuelas (docentes y expertos)}


Prácticas sociales en torno a la inclusión de la discapacidad sensorial en escuelas públicas de Copiapó de Chile

En relación a las prácticas sociales inclusivas de las escuelas, el concepto de inclusión es mayormente vinculado con brindar acceso de ingreso físico al establecimiento educacional e infraestructura del mismo. Por otra parte, la inclusión también se vincula con las adecuaciones necesarias realizadas y/o esperadas para que los estudiantes con NEE puedan desenvolverse libremente y con calidad en la comunidad escolar a lo largo de su proceso formativo. En un sentido más amplio, la inclusión es vista por los informantes como una forma de entregar herramientas por parte del PIE a los estudiantes. La siguiente cita da cuenta de estas necesidades de inclusión:

Por ejemplo, a xxx le grababan las pruebas, las evaluaciones, él las respondía, ningún problema, porque ese programa le va diciendo y le va leyendo y él va respondiendo y también está escuchando si la respuesta que él está dando está bien, pero va a llegar un momento dado en que no tenga energía para cargar el netbook, no puede, en cambio él va con su regleta y toma apuntes, o una grabadora. En fin, hasta cierto modo la tecnología es importante para ellos. (Docente, entrevista 1)

La aceptación del estudiante se relaciona con la implementación de técnicas de enseñanza y de las actividades recreativas que los docentes realizan. Respecto a la adaptación metodológica se encuentra como práctica inclusiva el trabajo de docentes apoyados por los profesionales (no docentes) con los que cuenta el establecimiento. La creatividad e innovación del docente se da a conocer en las evaluaciones diferenciadas y en la ejecución de talleres donde contar con los recursos tecnológicos para los estudiantes se hace indispensable. Es lo que se presenta en el siguiente testimonio:

Bueno, primero hay que dejar en claro que el concepto de inclusión se trata de que nosotros como sociedad y, en este caso, como docente especialista pueda hacer todas las adaptaciones que sean necesarias, en este caso mi alumno que tiene una discapacidad visual se sienta integrado al sistema educativo, yo hago la adaptación para que se sienta incluido. (Docente, entrevista 5) 
Prácticas sociales en torno a la inclusión de la discapacidad sensorial en escuelas públicas de Copiapó de Chile

A su vez el profesional de apoyo del PIE centra la atención en las necesidades del estudiante y en algunas ocasiones lo integra desde el aula a las actividades, ayudando al desarrollo de potencialidades y aprendizaje de la autonomía personal. Es lo que se expresa en la siguiente cita:

Se les hace adaptaciones a la metodología; en la forma se cambia, no en los contenidos. Eloísa no vino ahora pero es súper habilidosa, a ella no se le ha cambiado mucho el plan, solo las metodologías porque es muy habilosa y para ella el Ministerio manda los libros, todo. (Docente, entrevista 19)

En las observaciones realizadas en uno de los establecimientos educacionales se puede apreciar que una estudiante con discapacidad visual es llevada al aula de recursos y se le enseña el sistema de lectura braille.

En las prácticas inclusivas, se encuentra el acceso como parte de las adecuaciones de la comunidad educativa y de la acogida del establecimiento, donde en algunas ocasiones se encontró la proactividad de los docentes, pero que, en gran medida, se generaba por los compañeros que cuidaban de ellos. Esta apreciación queda manifestada claramente por una docente entrevistada que señaló lo siguiente:

Hay niños que saben, pero porque han sido lazarillos de ellos, entonces ahí me dicen -tía me enseña-, pero por iniciativa de ellos; o como los niños van con la regleta a la sala, también se motivan y eso es bueno. Igual todos los niños han buscado lazarillo y esos niños le ayudan a desenvolverse. (Docente, entrevista 19)

También en una de las observaciones realizadas se pudo pesquisar que "Ios compañeros ayudan a XXX a bajar la escalera en el recreo de las 9:30 h, la agarran del brazo y le van contando los peldaños de la escalera" (Cita diario de campo, establecimiento educacional).

La visión de inclusión que tienen los dispositivos educacionales es que comienza en la familia otorgándoles a los estudiantes aceptación y apoyo. También es vista como una forma de no discriminación y aceptación. El vigente currículo nacional de educación señala que el rol del docente va más allá de la 
Prácticas sociales en torno a la inclusión de la discapacidad sensorial en escuelas públicas de Copiapó de Chile

enseñanza y está asociado a la aceptación universal, sin importar la raza, cultura, etnia y religión que el estudiante o su familia pueda tener.

\section{Prácticas sociales en torno a la inclusión, según las familias}

Las familias (jóvenes y apoderados) entienden por inclusión a la apertura y acceso físico al establecimiento educacional, no importando si la escuela cuenta con los elementos físicos-técnicos en su interior (computadores, software, impresoras braille, etc.), y/o adecuaciones necesarias para el aprendizaje de sus hijos. Además, la inclusión es comprendida por las familias desde la carencia de dispositivos adecuados, que reciban a sus hijos, y que por lo tanto en este contexto es entendida como una iniciativa difícil de lograr. Es lo que se observa en la siguiente cita:

Yo creo que depende de cómo sea la persona, porque si usted es una persona que no tiene iniciativa propia y no es una persona sociable obviamente que el medio no va venir a usted a sociabilizar, porque uno igual tiene que ser movido y tiene que ser así y así y hago esto, entonces depende mucho de la persona cómo se va realizar la inclusión. (Apoderada, entrevista 13)

De las experiencias positivas que han vivenciado las familias en torno a la inclusión, se destaca que ven a sus hijos incluidos socialmente, lo que es causa de la colaboración del grupo familiar brindando apoyo. Las familias asocian el concepto de discapacidad con el de normalización, por lo que para ellos la discapacidad no es un impedimento para realizar las cosas. Por ejemplo, un participante señala que "en lo académico sí me he sentido integrado en el liceo en la media y en la universidad también. Sí me he sentido incluido" (Joven, entrevista 18).

Otra cita de una madre con un hijo con discapacidad visual señala: "Con xxx la discapacidad que él tiene es normal. Le he dicho de chiquitito que la 
Prácticas sociales en torno a la inclusión de la discapacidad sensorial en escuelas públicas de Copiapó de Chile

discapacidad visual que él tiene no es un impedimento para que pueda hacer las cosas y salir adelante" (Apoderada, entrevista 17).

Si bien la comprensión de la inclusividad se ve fuertemente vinculada a los elementos físicos y técnicos del establecimiento educacional, por otro lado, se observa también una visión por parte de las familias acerca de la inclusión como un derecho que tienen las personas con discapacidad y que, por lo tanto, deben acceder a una educación por derecho propio con el apoyo de los establecimientos educacionales y sus profesionales. Por ejemplo, una apoderada plantea lo siguiente: "Que entiendo, que esa parte, cuando apoyan a los niños que tienen algunos un pequeño retraso, una pequeña dificultad para aprender, yo entiendo integración de esa forma" (Apoderada, entrevista 15). Otra cita de una apoderada señala que: "Si podía salir adelante, acá con los obstáculos que se le habían dado y lo recibió la xxx, él era muy chico para irse y hemos salido adelante con él" (Apoderada, entrevista 17).

En relación a las prácticas no inclusivas de las familias, se plantea una contradicción con el municipio y se observa que hay dificultades en el ingreso y acceso fisico de los estudiantes con discapacidad sensorial a los establecimientos, sumado a la falta de recursos (técnicos, materiales y humanos) y a la mala administración de recursos económicos en Copiapó. Coincide el discurso de las familias y los establecimientos en que existen varias prácticas no inclusivas (ver más adelante, Tabla 4). De esta forma los resultados no dan cuenta de una exclusión completa, sino más bien de prácticas inclusivas que se vuelven no inclusivas.

Por último, otra categoría fundamental es la empatía reportada y observada con las familias por parte de los docentes, las cuales dan cuenta abiertamente del compromiso de ellos con la integración de sus hijos, facilitando esta constatación la adecuación mutua en el proceso formativo de los estudiantes. Se observó claramente que para las familias es importante y esencial la disposición del cuerpo directivo y docente con la enseñanza de sus hijos y sus necesidades; incluso 
Prácticas sociales en torno a la inclusión de la discapacidad sensorial en escuelas públicas de Copiapó de Chile

mucho más que el compromiso esperado de los profesionales del PIE (psicólogos, asistentes sociales, psicopedagogos, orientadores familiares).

\section{Prácticas sociales inclusivas (Estado, escuelas y familias)}

El Estado expresa que la inclusión es el ingreso, otorga el derecho a la educación y proporciona leyes que se relacionan con prácticas inclusivas de acceso universal, de mejoramiento en la calidad de vida, con compromiso en la equidad, lo que se observa en el código de adaptaciones de los programas educativos. De esta forma se relacionan las leyes con los decretos, buscando la igualdad de oportunidades, incluyendo las adaptaciones metodológicas, que se relacionan con el PIE.

Para las escuelas, donde se encuentran los docentes y expertos, la inclusión se entiende como el ingreso de los estudiantes con discapacidad sensorial a los establecimientos y en la no discriminación hacia los educandos. Tienen una visión de inclusión que implica ayudar a los estudiantes con discapacidad sensorial, con apoyo del PIE.

En el discurso y práctica de la escuela la entrega de apoyo es necesaria como acto inclusivo y esto es posible a través de herramientas técnicas y recursos humanos; es decir, contando con tecnología y profesionales. Se relaciona con la adecuación mutua de las escuelas y las familias.

Como apoyo a la inclusión, algunos expertos señalan la realización de actividades con los niños: "Por ejemplo ahí en cuanto a la inclusión de las escuelas especiales siempre se dan talleres o sea como actividades extra programáticas. Se lleva a los niños a que conozcan o se relacionen con otras entidades de la sociedad" (Experto, entrevista 2). Un ejemplo de práctica inclusiva, que se pesquisó en las observaciones, fue en una escuela donde se contaba con la implementación y uso de tecnología.

El compañerismo de los pares hacia los estudiantes con discapacidad sensorial es de gran importancia, fundamentalmente porque se hacen parte del 
Prácticas sociales en torno a la inclusión de la discapacidad sensorial en escuelas públicas de Copiapó de Chile

aprendizaje de los estudiantes con NEE y, en varias ocasiones, según la triangulación de la información, se obtuvieron resultados que daban cuenta de que los compañeros no solo ayudan en las tareas, sino que también dictan las materias, manteniendo una participación activa en la colaboración con las distintas evaluaciones que se les realiza a los estudiantes con discapacidad sensorial.

\section{Prácticas sociales no inclusivas, según las familias (jóvenes y apoderados)}

Las familias consideran prácticas sociales no inclusivas al retraso y desconfianza en las políticas públicas, a la existencia de discriminación en la educación básica y media, a la visión de inclusión como sinónimo de discriminación por parte de las familias, a los PIE, a la precaria administración de recursos económicos, a las dificultades para que los niños ingresen a los establecimientos, al desconocimiento de la Ley de inclusión y a la escasa oportunidad de la comuna. La siguiente cita da cuenta de la discriminación percibida por las familias:

Después fue la hazaña para empezar a buscar liceo. Fue muy difícil para nosotros eso, porque ella la opción que tenía era el colegio xxx porque ahí había un niño ciego, pero ella no quería eso, porque estaba catalogado como un mal colegio. Los colegios católicos fueron más discriminatorios que los demás colegios. (Apoderada, entrevista 11)

Se observa como práctica no inclusiva la discriminación por parte del Ministerio, donde falta adaptación de los contenidos de las evaluaciones estandarizadas nacionales al sistema braille. Por lo mismo se estaría discriminando en las evaluaciones SIMCE y PSU (Prueba de Selección Universitaria), lo que genera frustración del estudiante con discapacidad y su familia, debido a que el compromiso de oportunidades no se encuentra realizado en la práctica.

Las familias y jóvenes plantean que para estar preparados para la inclusión se necesita de los PIE, sin embargo, existen establecimientos que carecen de elementos, y que además el PIE en la educación secundaria apoyaría a los 
Prácticas sociales en torno a la inclusión de la discapacidad sensorial en escuelas públicas de Copiapó de Chile

estudiantes que tienen pequeños retrasos en el aprendizaje, dejando afuera otros diagnósticos importantes.

Los establecimientos educativos discriminan y cuesta que reciban a los niños con discapacidad sensorial. Como explica una apoderada en el siguiente comentario:

Él era muy chico para irse y hemos salido adelante. De primero me dijeron que no, que no me lo podían recibir y nos mandaron al tiro para Serena, y para nosotros era muy lejos irnos para allá y tampoco no lo íbamos a dejar ir, estaba muy chiquitito. (Apoderada, entrevista 23)

No solamente hay dificultades en el ingreso a los establecimientos, sino que también se visualiza que el acceso físico es dificultoso, existiendo falta de dispositivos especiales (alarmas de luces, señalizaciones, etc.) lo que da cuenta de una deficiencia en la infraestructura. Además existe un desconocimiento del trato a personas con discapacidad, lo que lleva al cuestionamiento de la inclusión por parte de las familias y jóvenes con discapacidad sensorial.

Por último, las familias y los propios usuarios hacen referencia a que los PIE presentan irregularidades y, en vez de ser algo beneficioso, se convierten en una palabra más que carece de una práctica, como debería serlo, pues además los estudiantes de educación secundaria no son considerados en ellos, debido a que solo un establecimiento en la ciudad de Copiapó imparte esta ayuda a los adolescentes.

\section{Tablas sinópticas}

A continuación se presentan las síntesis de las familias semánticas obtenidas tras la codificación axial.

Tabla 1. Similitudes de prácticas sociales inclusivas

\begin{tabular}{lll}
\hline Estado (Leyes y Decretos) & Escuela & Familia \\
\hline Visión centrada en otorgar & Visión centrada en otorgar & Visión centrada en otorgar \\
ingreso a los estudiantes. & ingreso a los estudiantes. & ingreso a los estudiantes.
\end{tabular}


Prácticas sociales en torno a la inclusión de la discapacidad sensorial en escuelas públicas de Copiapó de Chile

Se promueven los proyectos de integración y la entrega de herramientas (recursos).

Acerca a la red familiar al proceso de aprendizaje de los estudiantes.
Se promueve el trabajo con proyectos de integración.

Promover el apoyo familiar en la enseñanza.
Se preocupa de que trabajen con proyectos de integración los establecimientos.

Apoyo familiar (familia capacitada).

Fuente: Elaboración propia.

Tabla 2. Diferencias de prácticas sociales inclusivas

\begin{tabular}{|c|c|c|}
\hline Estado (Leyes y Decretos) & Escuela & Familia \\
\hline $\begin{array}{c}\text { Visión centrada en el ingreso } \\
\text { de los estudiantes. }\end{array}$ & $\begin{array}{c}\text { Visión centrada en el ingreso } \\
\text { de los estudiantes. }\end{array}$ & $\begin{array}{l}\text { Visión centrada en el ingreso } \\
\text { de los estudiantes y acceso } \\
\text { físico en los establecimientos. }\end{array}$ \\
\hline $\begin{array}{l}\text { Se promueven los proyectos } \\
\text { de integración y la entrega de } \\
\text { herramientas (recursos). }\end{array}$ & $\begin{array}{l}\text { Se promueve el trabajo con } \\
\text { proyecto de integración. }\end{array}$ & $\begin{array}{l}\text { La proactividad del docente es } \\
\text { más importante que los } \\
\text { proyectos de integración para } \\
\text { las familias. }\end{array}$ \\
\hline $\begin{array}{l}\text { Se realizan planes y decretos } \\
\text { con adaptaciones curriculares. }\end{array}$ & $\begin{array}{c}\text { Se promueven las } \\
\text { adaptaciones curriculares. }\end{array}$ & $\begin{array}{l}\text { Las adaptaciones curriculares } \\
\text { son importantes para las } \\
\text { familias como práctica inclusiva } \\
\text { pero predomina la metodología } \\
\text { del docente. }\end{array}$ \\
\hline
\end{tabular}

Fuente: Elaboración propia.

Tabla 3. Similitudes de prácticas sociales no inclusivas

\begin{tabular}{ccc}
\hline Estado (Leyes y Decretos) & Escuela & Familia \\
\hline Escasa promoción de la nueva & Ley de discapacidad poco & Ley de discapacidad poco \\
Ley de discapacidad 20.422. & conocida. & conocida. \\
Escasez de supervisión de los & Irregularidades en los & Irregularidades en los \\
programas implementados. & proyectos de integración. & proyectos de integración.
\end{tabular}


Prácticas sociales en torno a la inclusión de la discapacidad sensorial en escuelas públicas de Copiapó de Chile

No se estipula en la Ley

20.422 infraestructura en los

establecimientos para

discapacidad sensorial.
Acceso físico e ingreso

dificultoso en los

establecimientos para los

estudiantes con discapacidad

sensorial.
Acceso físico e ingreso

dificultoso en los

establecimientos para los

estudiantes con discapacidad

sensorial.

Fuente: Elaboración propia.

Tabla 4. Diferencias de prácticas sociales no inclusivas

\begin{tabular}{|c|c|c|}
\hline Estado (Leyes y Decretos) & Escuela & Familia \\
\hline $\begin{array}{l}\text { Irregularidades en la entrega } \\
\text { de herramientas y recursos } \\
\text { para los establecimientos y } \\
\text { familias de niños con } \\
\text { discapacidad. }\end{array}$ & $\begin{array}{c}\text { Falta de recursos y } \\
\text { capacitaciones para los } \\
\text { docentes. }\end{array}$ & $\begin{array}{l}\text { Escasez de recursos técnicos y } \\
\text { materiales entregados por el } \\
\text { estado y escuela para las } \\
\text { familias de estudiantes con } \\
\text { discapacidad. }\end{array}$ \\
\hline $\begin{array}{c}\text { Vigencia de Ley antigua } \\
19.284 .\end{array}$ & $\begin{array}{c}\text { Desconocimiento de los } \\
\text { docentes sobre la vigencia de } \\
\text { la Ley } 19.284 \text {. }\end{array}$ & $\begin{array}{c}\text { Desconocimiento, retraso y } \\
\text { desconfianza hacia las políticas } \\
\text { públicas. }\end{array}$ \\
\hline $\begin{array}{l}\text { El Estado habla de inclusión y } \\
\text { se rige por la Ley antigua que } \\
\text { establece decretos de } \\
\text { integración. }\end{array}$ & $\begin{array}{c}\text { Pruebas de selección para } \\
\text { ingresar a los establecimientos. }\end{array}$ & $\begin{array}{l}\text { Discriminación por parte de } \\
\text { algunos profesionales. }\end{array}$ \\
\hline
\end{tabular}

Fuente: Elaboración propia.

\section{DISCUSIÓN Y CONCLUSIONES}

Tras el análisis de la información que entregaron los informantes claves y el análisis de documentos afines, se desprende que lo declarado por el Estado se contrapone en parte con su práctica diaria en los establecimientos educacionales chilenos, particularmente en lo observado en la comuna de Copiapó. Se constató que lo estipulado en la № 20.422 (Ministerio de Planificación, 2010), respecto de las normas sobre la igualdad de oportunidades e inclusión social de personas con discapacidad, no se encuentra amplia e igualitariamente implementada en todos los establecimientos educacionales de la ciudad, dejando un gran vacío en la 
Prácticas sociales en torno a la inclusión de la discapacidad sensorial en escuelas públicas de Copiapó de Chile

necesidad real de los estudiantes con NEE de la región. Si bien se señala que "El Estado garantizará a las personas con discapacidad el acceso -según corresponda- a los establecimientos públicos y privados del sistema de educación regular o a los establecimientos de educación especial, que reciban subvenciones o aportes del Estado" (Ministerio de Planificación, 2010, p. 19); no existen dispositivos educativos adaptados a las necesidades de los niños con discapacidad sensorial en los establecimientos educacionales de la comuna, nuevamente mostrando un vacío entre lo que se declara y lo que se hace en la realidad.

Las familias entrevistadas, conformadas por jóvenes y apoderados, entregaron escasa información sobre las prácticas inclusivas en sus establecimientos de origen, destacando dentro de esta escasez de información la falta de recursos y conformismo referente a la educación entregada en los dispositivos de educación de la comuna. En este sentido, los informantes (familias y escuelas) comparten que la inclusión comprende básicamente el acceso físico a los establecimientos educacionales, independientemente de si estos cuentan o no con PIE acordes a las necesidades educativas especiales de sus estudiantes. Considerando estos datos, la inversión pública en recursos materiales es necesaria para que estudiantes con NEE en la región de Atacama tengan un mejor acceso a un proceso educativo adaptado a sus necesidades y de calidad. Si bien esto es solo una dimensión del concepto de inclusión, permite, a pesar de su comprensión limitada, plantear que es necesaria la incorporación de elementos tecnológicos en las aulas para facilitar el aprendizaje y desarrollo de las habilidades que estos niños presentan.

No obstante, estas observaciones denotan un déficit en términos de la comprensión del concepto de inclusión. Esto se explicaría por la falta de difusión por parte de las autoridades regionales, en contraposición a la de otro tipo de temáticas como la violencia de género o la preferencia y/u orientación sexual. Esta situación queda al descubierto en el discurso de los docentes, quienes entienden la inclusión como una "ayuda", evidenciándose claramente una confusión entre los 
Prácticas sociales en torno a la inclusión de la discapacidad sensorial en escuelas públicas de Copiapó de Chile

términos de integración e inclusión, predominando el primero por sobre el segundo. Por otra parte, las familias, en la necesidad de matricular a sus hijos en el establecimiento educacional de su agrado (por proximidad física y ahorro en el traslado) asumen un rol de displicencia y pasividad, lo que genera en las escuelas el desconocimiento de la situación real de los educandos en términos de sus NEE, muchas veces ocultas por la propia familia. Mencionan también que algunos establecimientos educacionales muestran preferencia por estudiantes que no poseen NEE, situación que fomenta el desconocimiento por parte de las familias de las posibilidades de atención para sus hijos y, al mismo tiempo, fomenta prácticas no inclusivas -y finalmente- de discriminación.

Un punto a destacar es la no existencia de profesionales especializados en las escuelas en temáticas de discapacidad, generando desigualdad y acciones fallidas de inclusión educacional que pueden, en algún momento, perjudicar el desarrollo pedagógico de los niños con NEE, creando mayores brechas entre lo que se estipula en la Ley 20.422 ((Ministerio de Planificación, 2010) y lo que ofrecen los establecimientos educacionales. Por ejemplo, la mayoría de los establecimientos de la región de Atacama no poseen adaptaciones curriculares especiales para estudiantes con discapacidad auditiva y visual como son señaladas en el decreto $N^{\circ} 86$ y № 89 del MINEDUC (1990). Los docentes afirmaron que evalúan las NEE de sus estudiantes de la forma que estiman conveniente en un momento dado, sin un juicio técnico, con el peligro de realizar una evaluación no apropiada. Por ejemplo, las familias manifiestan en sus discursos la falta de educación sobre el lenguaje de señas en la comunidad educativa, discurso que se repite en los mismos docentes, haciendo alusión a la escasa capacitación que reciben en esta herramienta para los casos de estudiantes sordos.

De acuerdo a la información recolectada, se puede concluir que en la ciudad de Copiapó existe un conformismo cultural en las familias y las escuelas a las que asisten niños con discapacidad visual y auditiva, el cual se produciría por la escasa oferta de establecimientos que cuentan con especialistas que apoyen 
Prácticas sociales en torno a la inclusión de la discapacidad sensorial en escuelas públicas de Copiapó de Chile

pedagógicamente a los niños con NEE; y también debido a la desinformación que prima sobre el tema. Según el discurso de las familias, éstas no asumen la discapacidad de sus hijos, tienden a verlos sin tal condición. Por otra parte, se observó que los docentes también realizan giros lingüísticos en desmedro de la condición real de niños con NEE, por ejemplo, realizando comparaciones entre la discapacidad del estudiante con su inteligencia, en menoscabo de la primera.

En síntesis, las familias en su intento de no reconocer la condición de sus hijos, y los docentes al ver la discapacidad como un déficit intelectual del estudiante, mantienen prácticas no inclusivas y discriminadoras a pesar del discurso social que manejan. Es de vital importancia relevar en este punto para la aminoración de las problemáticas detectadas en este estudio, la apertura manifestada por algunos de los actores claves en relación a la necesidad de adaptaciones curriculares, trato igualitario, junto a los recursos necesarios para ingresar y permanecer en el establecimiento educacional y en el proceso educativo.

Otro aspecto a destacar es la falta de capacitación resaltada por los docentes para trabajar con estudiantes con discapacidad. Esto se observó en el desconocimiento que tienen respecto de la discapacidad sensorial en lo general y en lo particular, por ejemplo en la falta de capacitación en el lenguaje de señas. Se puede concluir que los establecimientos educacionales de la comuna de Copiapó y por extensión a toda la región de Atacama, no se encuentran profesionalmente preparados amplia y cabalmente para recibir y trabajar con estudiantes con NEE. Además, la mayoría de estos establecimientos no realiza prácticas inclusivas orientadas a talleres de menor duración para la capacitación y sensibilización en el trato y trabajo pedagógico especializado con estudiantes con discapacidad. Tampoco se evidenciaron prácticas educativas de promoción de la inclusividad en la totalidad de la comunidad educativa por su propia iniciativa, a través de charlas, jornadas, ferias, poster y similares. Debido a lo anterior, este estudio concluye que no existe una cultura de la enseñanza sobre el significado de la discapacidad 
Prácticas sociales en torno a la inclusión de la discapacidad sensorial en escuelas públicas de Copiapó de Chile

sensorial a nivel docente y de los profesionales de la educación, ni de la promoción y educación de la familia y la comunidad.

Dentro de las prácticas inclusivas que se destacan en el presente estudio se encuentra la preocupación por el acceso físico de los estudiantes con discapacidad a los establecimientos y la creación de material por parte de los docentes. Los pares también juegan un papel importante a la hora de ayudar a otros estudiantes con discapacidad, colaborando en la sala de clases junto a las instrucciones e indicaciones dadas por sus docentes, y muchas veces por iniciativa propia -por ejemplo- facilitándoles a sus compañeros ayuda durante los recreos, guiándolos o haciéndoles compañía, conversando y/o jugando con ellos.

Tras el análisis de la información se desprende que las prácticas sociales que se implementan en los sistemas educativos de la ciudad de Copiapó tienen significancia hacia la apertura y la aceptación de los educandos en el establecimiento, donde se observó la incorporación de las políticas públicas respecto a la inclusión de los estudiantes con discapacidad sensorial. Los resultados permiten hipotetizar que existe una mirada conformista, pasiva y con escasa proactividad con respecto a la inclusión educativa desde las prácticas inclusivas de la escuela y la familia. Dentro de las escuelas la inclusión se expresa a través del ingreso, sin mayor dificultad, al establecimiento; sin embargo, una vez dentro no se asegura su permanencia con la atención especializada adecuada y los recursos necesarios para un óptimo desarrollo. Es importante destacar que los actores entrevistados concuerdan que, si bien han mejorado las políticas públicas en educación, todavía falta que exista integración en los dispositivos, no solamente en la comuna, sino a nivel de todo el país.

Las interrogantes que generó esta investigación pueden servir como líneas de investigación que orienten futuros estudios hacia el análisis del estado actual y las proyecciones existentes en Copiapó en torno a la atención de los estudiantes con discapacidad sensorial. Una de las primeras preguntas que surgen se relaciona con las NEE: ¿Cuál es el impacto que generan los PIE en la población con discapacidad sensorial? Esta pregunta surge por las irregularidades 
Prácticas sociales en torno a la inclusión de la discapacidad sensorial en escuelas públicas de Copiapó de Chile

observadas en las prácticas de implementación de los PIE. Una segunda pregunta que nace en este proceso investigativo tiene relación con la información obtenida de las entrevistas: ¿A qué se debe la escasez de profesionales capacitados en el área de educación especial? Los informantes claves, en reiteradas ocasiones, dieron cuenta de que la mayoría de los docentes no se encuentran capacitados en la educación especial, haciendo alusión a que los estudiantes se encuentran carentes de apoyo de profesionales especializados. La tercera interrogante señala: ¿Por qué en la comuna no existe un catastro de los usuarios? Los informantes, en sus experiencias con las prácticas, afirman que hay un desconocimiento del número de estudiantes con discapacidad. Por último, y una de las preguntas más interesantes, tiene relación con los usuarios y las familias: ¿Qué lleva a que los usuarios y familias tengan una postura pasiva y poco informada sobre las prácticas que establece el Estado de Chile en la educación? Se considera esta última pregunta como una paradoja debido a que, a pesar de la existencia de una ley nueva, se mantiene en uso la ley antigua: ¿A qué se debe que el MINEDUC actualmente trabaje con la Ley 19.284 (Ministerio de Planificación y Cooperación, 1994)? Todas las interrogantes expuestas dejan abierto el campo de estudio a futuras investigaciones en el ámbito social educativo en la comuna de Copiapó.

\section{REFERENCIAS}

Ainscow, M. (2005). Developing inclusive education systems: what are the levers for change? Journal of Educational Change 6, 109-124.

Camacho-Ríos, A. (2011). Socio epistemología y prácticas sociales. Hacia una enseñanza dinámica del cálculo diferencial. Revista Iberoamericana de Educación Superior, vol. II, núm. 3, 152-171. Instituto de Investigaciones sobre la Universidad y la Educación. Estado de México: México.

Castillo, M. (2013). Educación municipal: Crónicas de irreguaridades anunciadas. Atacama Viva. Recuperado el 19 de Febrero de 2014 de: 
Prácticas sociales en torno a la inclusión de la discapacidad sensorial en escuelas públicas de Copiapó de Chile

http://www.supereduc.cl/sala-de-prensa/superintendencia-en-la-prensa/700en-la-prensa-2013/en-la-prensa-2013-febrero.html

Charmaz, K. (2007). Constructing Grounded Theory: A Practical Guide through Qualitative Analysis (Introducing Qualitative Methods series). Sage Publications.

Delors, J. et al. (1996). La educación encierra un tesoro. Informe a la UNESCO de la Comisión Internacional sobre la Educación para el siglo XXI. París: UNESCO. Recuperado el 14 de enero de 2015 de: http://www.unesco.org/education/pdf/DELORS_S.PDF

Egea, C., \& Sarabia, A. (2001). Clasificaciones de la OMS sobre discapacidad. Boletín del Real Patronato, (50), 15-30.

Fundación Chile. (2013). Implementación de programas escolares en establecimientos educacionales con estudiantes con necesidades educativas especiales transitorias. Santiago: Chile. Recuperado de http://portales.mineduc.cl/usuarios/edu.especial/doc/201402101720120.Res umenEstudiolmplementacionPIE2013.pdf

García R., J. (2011). Modelo educativo basado en competencias: importancia y necesidad. Revista Electrónica Actualidades Investigativas en Educación Volumen 11, Número 3, 1-24.

Garnique-Castro, F. \& Gutiérrez-Vidrio, S. (2012). Educación básica e inclusión: un estudio de representaciones sociales. magis, Revista Internacional de Investigación en Educación, 4 (9), 577-593.

Granada Azcarraga, M; Pomes Correa, M.P. \& Sanhueza Henríquez, S. (2013). Actitud de los profesores hacia la inclusión educativa. Pap. trab. - Cent. Estud. Interdiscip. Etnolingüíst. Antropol. Sociocult. [online]. n. 25. 51-59.

Guerra, T; Arcos, N; Riquelme, G; Ramírez, P; \& Guzmán, J. (2012). Ley SEP: Ranking de las 20 municipalidades más cuestionadas por Contraloría. Recuperado el 12 de abril de 2014 de: http://ciperchile.cl/2012/05/28/leysep-ranking-de-la-20-municipalidades-mas-cuestionadas-por-contraloria/ 
Prácticas sociales en torno a la inclusión de la discapacidad sensorial en escuelas públicas de Copiapó de Chile

Íniguez, L. (1999). Investigación y evaluación cualitativa: bases teóricas y conceptuales. Aten Primaria. ํㅡㄴ 23, 496-502.

Larraguibel Quiroz, E. (2012). La inclusión en la educación parvularia. Revista docencia, 81-87. Santiago de Chile.

Lissi, M. R.; Zuzulich, M.S.; Hojas, A.M.; Achiardi, C.; Salinas, M. \& Vasquez, A. (2014). En el camino hacia la educación superior inclusiva en chile: Fundamentos y adecuaciones curriculares para estudiantes con discapacidad sensorial o motora. Dirección de Asuntos Estudiantiles. Salud Estudiantil. Programa para la Inclusión de Alumnos con Necesidades Especiales PIANE-UC.

Marchesi, A., Coll, C., y Palacios, J. (2012). Desarrollo Psicológico y Educación. Alianza Editorial. Madrid. Madrid: España.

Ministerio de Planificación y Cooperación (1994). Ley 19.284: Establece Normas para la Plena Integración Social de Personas con Discapacidad. Recuperado el 12 de enero de 2015 de: https://www.leychile.cl/Navegar?idNorma=30651

Ministerio de Planificación. (2010). Ley 20.422. Establece Normas Sobre Igualdad de Oportunidades e Inclusión Social de Personas con Discapacidad. Recuperado el 12 de enero de 2015 de: http://www.leychile.cl/Navegar?idLey=20422

Ministerio de Educación. (1998). Reglamenta Capitulo II Título IV de la ley n⒚284 que establece normas para la integración social de personas con discapacidad. Recuperado el 12 de enero de 2015 de: http://www.comunidadescolar.cl/marco_legal/Decretos/Decreto\%201\%20Re glamenta\%20Plena\%20Integracion.pdf

Ministerio de Educación Pública. (1990). Aprueba planes y programas de estudio para educandos con déficit visual. República de Chile. Recuperado el 20 de octubre de 2014 de: http://portales.mineduc.cl/usuarios/edu.especial/doc/201304231642460.Dec retoN89.pdf 
Prácticas sociales en torno a la inclusión de la discapacidad sensorial en escuelas públicas de Copiapó de Chile

Ministerio de Educación Pública. (1990). Aprueba planes y programas de estudio para atender niños con trastornos de la comunicación. República de Chile. Recuperado el 20 de octubre de 2014 de: http://portales.mineduc.cl/usuarios/edu.especial/doc/201304051153150.Dec retoN86.pdf

Ocampo, A. (2011). Inclusión de estudiantes en situación de discapacidad a la educación superior. Desafíos y oportunidades. Revista Latinoamericana de Educación Inclusiva. Vol. 6, № 2, 227-239. Santiago: Chile.

OMS. (2011). Resumen Informe Mundial Sobre La Discapacidad. Organización Mundial de la Salud y Banco Mundial. Impreso en Malta, Malta. Recuperado el 15 de noviembre de 2014 de: http://www.who.int/disabilities/world_report/2011/summary_es.pdf

ONU. (1959). Declaración de los Derechos del Niño. Recuperado el 24 de septiembre de 2014 de: http://www.unicef.org/argentina/spanish/7.Convencionsobrelosderechos.pdf

Romero, M; Kitaoka, E; Rodríguez, C. (2014). En la inclusión educativa, más allá de la pobreza y las etnias, están las personas con discapacidad. Revista Iberoamericana de Producción Académica y Gestión Educativa. Publicación \# 02 julio-diciembre. Recuperado el 20 de enero de 2015 de: http://www.pag.org.mx/index.php/PAG/article/view/141

Servicio Nacional de la discapacidad. (2013). Política Nacional para la Inclusión Social de las Personas con Discapacidad 2013-20120. Santiago. Chile. Recuperado el 26 de septiembre de 2014 de: http://www.senadis.gob.cl/descarga/i/1238

Sistema de Medición de la Calidad de la Educación (SIMCE). (2011). Informe de Resultados Nacionales. Ministerio de Educación. Unidad de Currículum y Evaluación SIMCE. Recuperado el 15 de abril de 2014 de: http://www.agenciaeducacion.cl/wpcontent/files_mf/informenacionalderesult adossimce2010247mb.pdf 
Prácticas sociales en torno a la inclusión de la discapacidad sensorial en escuelas públicas de Copiapó de Chile

Strauss, A. y Corbin, J. (2002). Bases de la investigación cualitativa. Técnicas y procedimientos para desarrollar la teoría fundamentada, pp. 110-154. Medellín, Colombia.

Torres, A.M. (2013). Las oportunidades de la integración educativa al ingreso de la educación regular según el decreto 170. Perspectiva Educacional, Vol.52, no1, 124-146. Valparaíso: Chile.

Trinidad, A., Carrero, V. \& R. Soriano (2006), Teoría fundamentada "Grounded Theory". La construcción de la teoría a través del análisis interpretacional. Cuaderno metodológico 37, CIS, Madrid: España.

Vigo, B., \& Soriano, B. (2014). Prácticas de enseñanza y aprendizaje creativo para una educación inclusiva en la formación inicial del profesor. Experiencias de investigación. Revista nacional e internacional de educación inclusiva, 7 (1), 30-45.

Recibido el 18 de febrero de 2016

Revisado el 7 de mayo de 2016 Aceptado el 12 de mayo de 2016 